\title{
Enquête pilote de surveillance biologique et comportementale sur les infections à VIH ou d'autres infections transmissibles par le sang menée auprès des Autochtones de Regina, en Saskatchewan
}

\author{
Tarasuk $\mathbf{J}^{1 *}$, Ogunnaike-Cooke $\mathbf{S}^{1}$, Archibald $\mathrm{C}^{1}$, Poitras $\mathbf{M}^{2}$, Hennink $\mathbf{M}^{3}$, Lloyd $\mathrm{K}^{3}$, Faye $\mathbf{R}^{3}$, Abbas $\mathbf{Z}^{3}$, Bourassa \\ $C^{4}$, Masching $R^{5}$, Bennett $R^{1}$, MacLean $R^{1}$, Malloch $L^{1}$, Kim $J^{1}$ \\ 1. Centre de la lutte contre les maladies transmissibles et les infections, Agence de la santé publique du Canada, Ottawa (Ontario) \\ ${ }^{2}$ Réseau All Nations Hope AIDS Network, Regina (Saskatchewan) \\ ${ }^{3}$ Regina Qu'Appelle Health Region, Regina (Saskatchewan) \\ ${ }^{4}$ Université des Premières nations du Canada, Regina (Saskatchewan) \\ ${ }^{5}$ Réseau canadien autochtone du sida, Dartmouth (Nouvelle-Écosse) \\ ${ }^{*}$ Correspondance: jill.tarasuk@phac-aspc.gc.ca
}

\section{Résumé}

Contexte : Les Autochtones sont touchés par le VIH et d'autres infections transmissibles par le sang de manière disproportionnée. A-Track est un système de surveillance national de santé publique conçu pour surveiller le VIH et les infections connexes ainsi que les comportements et les facteurs sociodémographiques parmi les populations autochtones au Canada. L'enquête pilote portant sur le système de surveillance A-Track, la première en son genre au Canada, a été menée à Regina, en Saskatchewan, et a été mise en œuvre grâce à un partenariat lié à la santé publique et communautaire.

Objectif : Évaluer la prévalence du VIH, de l'hépatite $C$, de la syphilis ainsi que des facteurs sociodémographiques et des comportements à risque qui y sont associés chez les Autochtones de Regina, en Saskatchewan. L'enquête pilote avait pour objectif principal de fournir ces données de surveillance dans le but de prendre des mesures de santé publique et de déterminer si une activité de surveillance de la santé publique de ce type pourrait être réalisée dans un milieu urbain à l'échelle du pays.

Méthodologie : Les participants à l'enquête s'étaient autodéclarés Autochtones (Premières nations, Inuits ou Métis) ou revendiquaient une ascendance autochtone et étaient âgés de 16 à 60 ans. On a également demandé à ces personnes de fournir un échantillon de sang aux fins de dépistage des anticorps contre le $\mathrm{VIH}$, I'hépatite $\mathrm{C}$ et la syphilis. Des analyses descriptives ont été effectuées par le biais de comparaisons en fonction du sexe.

Résultats : 1064 personnes ont participé à cette enquête. L'âge moyen des participants était de 33 ans et $51 \%$ d'entre eux étaient de sexe masculin. La majorité des participants (93\%) vivait dans la partie urbaine de Regina au moment où l'enquête a été menée. Un peu plus de la moitié $(53,2 \%)$ des participants avaient été retirés de leur famille pendant l'enfance, $29,9 \%$ avaient habité dans un pensionnat pendant leur enfance et $57,7 \%$ avaient été placés dans un établissement correctionnel à un certain moment. Parmi les 1045 participants qui ont fourni un échantillon de sang suffisant aux fins du dépistage, 5,2\% ont été trouvés séropositifs pour le $\mathrm{VIH} ; 55,8 \%$ d'entre eux étaient au courant de leur séropositivité pour le VIH. L'exposition à vie à l'hépatite $\mathrm{C}$ était de $41,6 \%$; les proportions étaient beaucoup plus élevées chez les hommes que chez les femmes qui ont eu des résultats positifs au test de dépistage de l'exposition à I'hépatite C. La séroprévalence de la syphilis était très faible $(<1 \%)$. Près des trois quarts $(71,5 \%)$ des participants ont signalé avoir subi un test de dépistage du VIH au moins une fois dans leur vie. Parmi ceux-ci, $67,6 \%$ avaient subi un test pendant les 12 mois précédant l'entrevue.

Conclusion : Les Autochtones sont affectés de manière disproportionnée par l'épidémie de VIH/sida au Canada. Les résultats de l'enquête pilote $A$-Track peuvent être utilisés pour guider et évaluer les services de prévention et de traitement du VIH et d'autres infections connexes pour les Autochtones. Les leçons tirées de l'enquête pilote pourraient également être utilisées pour orienter la mise en œuvre possible du système A-Track dans d'autres sites urbains ou réserves au Canada. 


\section{Introduction}

Au Canada, les Autochtones sont touchés par le VIH/sida de manière disproportionnée. On estime qu'en 2011, 12,2 \% des nouvelles infections au VIH touchaient les Autochtones (1). À la fin de 2011, ces derniers représentaient 8,9\% des personnes vivant avec le VIH au Canada (1). À titre de comparaison, les Autochtones représentaient $4,3 \%$ de la population canadienne lors du recensement de 2011 (2).

Le système A-Track est un système de surveillance comportementale et biologique conçu pour surveiller la prévalence du VIH et des infections connexes ainsi que les facteurs sociodémographiques et les comportements à risque qui y sont associés parmi les populations autochtones au Canada. Le système A-Track a été testé à Regina, en Saskatchewan, de 2011 à 2012. L'enquête pilote avait pour objectif principal de fournir d'importantes données de surveillance et de déterminer si une activité de surveillance de la santé publique de ce type pourrait être réalisée dans un milieu urbain au Canada.

Le présent rapport contient certains résultats de l'enquête pilote A-Track et est un résumé d'un rapport plus approfondi intitulé Résumé des principaux résultats de l'enquête pilote A-Track, 2011-2012 (3).

\section{Méthodologie}

Le système A-Track est un système de surveillance comportementale et biologique qui contrôle la prévalence du VIH et des infections connexes ainsi que les facteurs sociodémographiques et les comportements à risque qui y sont liés parmi les populations autochtones au Canada. Une enquête pilote a été menée à Regina, en Saskatchewan, du 5 décembre 2011 au 15 juin 2012.

L'enquête pilote A-Track a été élaborée et mise en œuvre par le biais d'un partenariat lié à la santé publique et communautaire. Un groupe consultatif communautaire, le réseau All Nations Hope AIDS Network, la région sanitaire de Regina Qu'Appelle, le Réseau canadien autochtone du sida, I'Université des Premières nations du Canada et l'Agence de la santé publique du Canada comptaient parmi les partenaires.

Le système de surveillance A-Track reconnaît le contrôle partagé des données des Autochtones, respecte les coutumes autochtones et est axé sur les principes du respect mutuel entre tous les intervenants, la reconnaissance d'une responsabilité partagée, la participation de la communauté autochtone et le recours à l'expertise locale existante. Le protocole du système de surveillance reconnaît les pratiques de recherches éthiques adaptées à la culture des Premières nations, des Inuits et des Métis et propres à leurs collectivités, y compris les principes de propriété, de contrôle, d'accès, de possession et de protection des données (4) et est conforme à l'Énoncé de politique des trois Conseils : Éthique de la recherche avec des êtres humains (5) et aux Lignes directrices pour la recherche en santé chez les peuples autochtones des Instituts de recherche en santé du Canada (6). Les données de l'enquête pilote menée à Regina sont gérées collectivement par le réseau All Nations Hope AIDS Network, la Regina Qu'Appelle Health Region et l'Agence de la santé publique du Canada.

La population cible de l'enquête pilote était des personnes qui se sont identifiées comme étant autochtones (membres des Premières nations, Inuits ou Métis) ou qui ont déclaré une ascendance autochtone et qui avaient entre 16 et 60 ans. La participation était volontaire, totalement anonyme et fondée sur un consentement verbal éclairé. Les participants ont été recrutés dans des organisations communautaires, des centres d'amitié, des points de services de soins de santé et d'autres lieux pertinents à Regina.

On a demandé à des participants consentants de répondre à un questionnaire portant sur des questions démographiques, le comportement sexuel, la consommation de drogues, les tests de dépistage du VIH et de l'hépatite C ainsi que les antécédents de traitement, l'accès aux services de santé et les connaissances sur le VIH. On a également demandé aux participants de fournir un échantillon de sang prélevé par ponction capillaire au bout du doigt qui a été analysé afin de dépister des anticorps contre le VIH, l'hépatite C et la syphilis.

Les données comprises dans ce rapport constituent les résultats descriptifs présentés pour l'échantillon global (ainsi que par sexe), afin de permettre les comparaisons entre les participants de sexe masculin et de sexe féminin en ce qui a trait aux caractéristiques démographiques, aux résultats du dépistage en laboratoire des anticorps, aux comportements sexuels et de consommation de drogues ainsi qu'au dépistage, aux soins et au traitement liés au VIH. Les résultats concernant les états d'infection au VIH, d'infection transmissible sexuellement et d'infection tuberculeuse autodéclarés, l'accès aux services de santé et les connaissances sur le VIH ne sont pas présentés ici, mais peuvent être obtenus dans 
le rapport complet (3). Les résultats provenant des données des tableaux dont les cellules comportent de faibles nombres doivent être interprétés avec prudence.

\section{Résultats}

Un total de 1064 individus ont participé à l'enquête pilote A-Track, dont deux ont déclaré une ascendance autochtone et 1062 se sont identifiés comme étant Autochtones (tableau 1). Parmi ces 1062 Autochtones, la majorité (90,1\%) s'est identifiée comme étant des membres des Premières nations. Un peu plus de la moitié $(50,7 \%)$ des participants étaient de sexe masculin et un peu moins de la moitié $(44,8 \%)$ des participants étaient âgés de 30 à 49 ans, avec une proportion légèrement plus faible (42,2\%) âgée de moins de 29 ans et de plus de 50 ans (13\%). La majorité des participants $(95,5 \%)$ ont déclaré leur orientation sexuelle comme étant hétérosexuelle, mais une proportion beaucoup plus élevée de femmes que d'hommes s'est identifiée comme étant gaie, lesbienne, bisexuelle ou autre (6,9\% par rapport à $2,2 \%$ ).

Tableau 1 : Caractéristiques démographiques des participants à l'enquête pilote A-Track

\begin{tabular}{|c|c|c|c|c|}
\hline $\begin{array}{c}\text { Caractéristiques démographiques et } \\
\text { expériences passées }\end{array}$ & $\begin{array}{c}\text { Total } \\
\text { ( } n=1064)\end{array}$ & $\begin{array}{l}\text { Hommes } \\
(n=539)\end{array}$ & $\begin{array}{l}\text { Femmes } \\
(n=525)\end{array}$ & Valeur $p$ \\
\hline $\begin{array}{r}\text { Sous-groupes autochtones }(\mathrm{n}=1062) \\
\text { Premières nations } \\
\text { Métis } \\
\text { Inuits }\end{array}$ & $\begin{array}{l}90,1 \%(957) \\
9,7 \%(103) \\
\quad<1 \%\end{array}$ & $\begin{array}{c}88,8 \%(477) \\
11 \%(59) \\
<1 \%\end{array}$ & $\begin{array}{l}91,4 \% \\
(480) \\
8,4 \%(44) \\
\quad<1 \%\end{array}$ & 0,357 \\
\hline $\begin{array}{r}\text { Âge en nombre d'années }(n=1064) \\
29 \text { et moins } \\
30 \text { à } 49 \\
50 \text { et plus }\end{array}$ & $\begin{array}{c}42,2 \%(449) \\
44,8 \%(477) \\
13 \%(138)\end{array}$ & $\begin{array}{c}37,5 \%(202) \\
48,2 \%(260) \\
14,3 \%(77)\end{array}$ & $\begin{array}{c}47,1 \% \\
(247) \\
41,3 \% \\
(217) \\
11,6 \%(61) \\
\end{array}$ & 0,007 \\
\hline $\begin{array}{r}\text { Orientation sexuelle }(n=1064) \\
\text { Gais, lesbiennes, bisexuels et autres } \\
\text { Hétérosexuels }\end{array}$ & $\begin{array}{c}4,5 \%(48) \\
95,5 \% \\
(1016) \\
\end{array}$ & $\begin{array}{c}2,2 \%(12) \\
97,8 \%(527)\end{array}$ & $\begin{array}{c}6,9 \%(36) \\
93,1 \% \\
(489) \\
\end{array}$ & $<0,001$ \\
\hline $\begin{array}{l}\text { Plus haut niveau de scolarité atteint } \\
\text { ( } \mathrm{n}=1 \text { 064) } \\
\text { Quelques années d'études secondaires ou } \\
\text { moins } \\
\text { Diplôme d'études secondaires } \\
\text { A réalisé des études supérieures }\end{array}$ & $\begin{array}{l}60,2 \%(640) \\
19,5 \%(208) \\
20,2 \%(214)\end{array}$ & $\begin{array}{l}60,3 \%(325) \\
20,2 \%(109) \\
19,3 \%(104)\end{array}$ & $\begin{array}{l}60 \%(315) \\
18,9 \%(99) \\
21 \%(110)\end{array}$ & 0,733 \\
\hline $\begin{array}{l}\begin{array}{l}\text { Revenu de l'ensemble des membres du } \\
\text { ménage }(n=738)^{1} \\
\text { Jusqu'à } 9999 \$ \\
10000 \$ \text { à } 39999 \$ \\
40000 \$ \text { et plus }\end{array}\end{array}$ & $\begin{array}{l}27,1 \%(200) \\
51,1 \%(377) \\
21,8 \%(161)\end{array}$ & $\begin{array}{c}27,7 \%(100) \\
46,3 \%(167) \\
26 \%(94)\end{array}$ & $\begin{array}{c}26,5 \% \\
(100) \\
55,7 \% \\
(210) \\
17,8 \%(67)\end{array}$ & 0,011 \\
\hline $\begin{array}{l}\text { Proportion de participants ayant été retirés } \\
\text { ou séparés de leur famille pendant l'enfance } \\
\text { par des agences de protection de l'enfance, } \\
\text { l'église ou des représentants }\end{array}$ & $53,2 \%(565)$ & $53 \%(285)$ & $\begin{array}{l}53,3 \% \\
(280)\end{array}$ & 0,907 \\
\hline
\end{tabular}




\begin{tabular}{|c|c|c|c|c|}
\hline gouvernementaux ( $n=1063$ ) & & & & \\
\hline $\begin{array}{l}\text { Proportion de participants ayant déjà } \\
\text { fréquenté un pensionnat pour enfants } \\
\text { autochtones pendant l'enfance }(n=1061)\end{array}$ & $29,9 \%(317)$ & $32,1 \%(172)$ & $\begin{array}{c}27,6 \% \\
(145)\end{array}$ & 0,112 \\
\hline $\begin{array}{l}\text { Proportion de participants ayant déjà été } \\
\text { placés en famille d'accueil pendant l'enfance } \\
\text { ( } n=1060)\end{array}$ & $43,4 \%(460)$ & $41,7 \%(223)$ & $\begin{array}{c}45,1 \% \\
(237)\end{array}$ & 0,256 \\
\hline $\begin{array}{l}\text { Situation de logement pendant les } 12 \text { mois } \\
\text { précédant l'entrevue }(n=1064)\end{array}$ & & & & \\
\hline 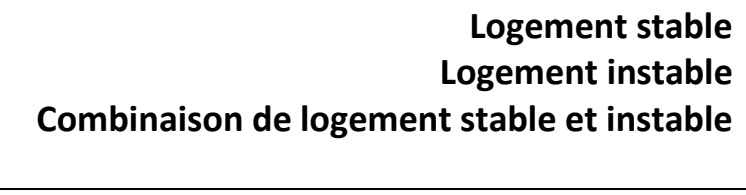 & $\begin{array}{l}73,5 \%(782) \\
6,4 \%(68) \\
20,1 \%(214)\end{array}$ & $\begin{array}{c}68,8 \%(371) \\
8,2 \%(44) \\
23 \%(124)\end{array}$ & $\begin{array}{c}78,3 \% \\
(411) \\
4,6 \%(24) \\
17,1 \%(90) \\
\end{array}$ & 0,001 \\
\hline $\begin{array}{l}\text { Proportion de participants ayant déménagé } \\
\text { au cours des } 12 \text { mois précédant l'entrevue, } \\
\text { peu importe la raison }(n=1064)\end{array}$ & $33,3 \%(354)$ & $33,2 \%(179)$ & $\begin{array}{c}33,3 \% \\
(175)\end{array}$ & 0,966 \\
\hline $\begin{array}{l}\text { Proportion de participants ayant déjà habité } \\
\text { dans un établissement correctionnel } \\
(n=1061)\end{array}$ & $57,7 \%(612)$ & $70,2 \%(376)$ & $45 \%(236)$ & $<0,001$ \\
\hline $\begin{array}{l}\text { Proportion de participants ayant habité dans } \\
\text { un établissement correctionnel pendant les } \\
12 \text { mois précédant l'entrevue ( } n=1064)\end{array}$ & $5,2 \%(55)$ & $7,2 \%(39)$ & $3,1 \%(16)$ & 0,002 \\
\hline
\end{tabular}

${ }^{1}$ Le revenu a été mesuré d'après le revenu de l'ensemble des membres du ménage, avant imposition et autres déductions, toutes sources confondues pour l'année se terminant le 31 décembre 2010.

${ }^{2}$ On a demandé aux participants d'indiquer tous les types d'endroits où ils avaient habité continuellement ou occasionnellement pendant les 12 mois précédant l'entrevue. Les réponses ont été classées selon les catégories suivantes : logement stable, logement instable et combinaison de logement stable et instable. Logement stable comprenait : vivre dans un appartement ou une maison ou l'appartement ou la maison d'un proche pendant les 12 mois précédant l'entrevue. Logement instable comprenait : vivre chez un ami, dans un hôtel ou un motel, dans une maison de chambres ou une pension de famille, dans un refuge ou une auberge, dans une maison de transition, dans un centre de désintoxication, dans un établissement correctionnel, dans un lieu public (comme une rue, des squats), dans un institut psychiatrique, dans un hôpital ou dans tout autre endroit jugé instable (p. ex. véhicule, tente, n'importe où à l'extérieur) dans les 12 mois précédant l'entrevue.

Un peu plus de la moitié $(51,1 \%)$ de tous les participants qui ont fourni des réponses à la question concernant le revenu du ménage ont déclaré que celui-ci se situait entre 10000 \& et 39000 \$. Des différences importantes ont été relevées entre les revenus déclarés des ménages des hommes par rapport à ceux des femmes. Plus de la moitié $(60,2 \%)$ des participants n'avaient pas terminé leurs études secondaires, 19,5\% avaient un diplôme d'études secondaires et 20,3\% avaient fait des études postsecondaires.

Un peu plus de la moitié $(53,2 \%)$ des participants avaient été retirés de leur famille pendant l'enfance, près d'un tiers $(29,9 \%)$ avaient habité dans un pensionnat pendant leur enfance et $43,4 \%$ avaient été placés en famille d'accueil pendant leur enfance. Aucune différence importante n'a été relevée entre les hommes et les femmes.

Bien que seulement $6,4 \%$ de tous les participants ont indiqué avoir vécu uniquement dans des logements instables pendant les 12 mois précédant l'entrevue, 20,1\% ont indiqué une combinaison de logement stable et instable. Une proportion significativement plus élevée d'hommes que de femmes ont déclaré un logement instable ainsi qu'une combinaison de logement stable et instable au cours des 12 mois précédant l'entrevue. Un tiers des participants (33,3\%) ont indiqué avoir déménagé pour une raison ou une autre au cours des 12 mois précédant l'entrevue; aucune différence importante n'a été relevée entre les hommes et les femmes.

Plus de la moitié de tous les participants $(57,7 \%)$ avaient, à un moment donné dans leur vie, habité dans un établissement correctionnel; cette proportion était beaucoup plus élevée chez les participants de sexe masculin que chez les participants de sexe féminin. La proportion d'hommes qui avaient vécu dans un établissement correctionnel au cours des 12 mois précédant l'entrevue était beaucoup plus élevée que la proportion de femmes (7,2\% par rapport à 3,1\%). 
Tableau 2 : Résultats des tests de dépistage du VIH, de I'hépatite C et de la syphilis pour les participants à l'enquête pilote A-Track

\begin{tabular}{|c|c|c|c|c|}
\hline Résultats de laboratoire & Total & Hommes & Femmes & Valeur $p$ \\
\hline \multicolumn{5}{|c|}{ Séroprévalence du VIH (parmi les participants ayant fourni un échantillon de sang, $n=1045$ ) $^{1}$} \\
\hline Participants séropositifs pour le VIH & $5,2 \%(54)$ & $6 \%(32)$ & $4,3 \%(22)$ & 0,213 \\
\hline $\begin{array}{r}\text { Proportion de participants séropositifs } \\
\text { pour le VIH ayant déclaré des antécédents } \\
\text { d'utilisation de drogues injectables ( } \\
=54)\end{array}$ & $92,6 \%(50)$ & $90,6 \%(29)$ & $95,5 \%(21)$ & $0,506^{5}$ \\
\hline $\begin{array}{l}\text { Proportion de participants séropositifs } \\
\text { pour le VIH qui étaient au courant de leur } \\
\text { séropositivité pour le VIH }(n=52)^{2}\end{array}$ & $55,8 \%(29)$ & $50 \%(15)$ & $63,6 \%(14)$ & 0,328 \\
\hline \multicolumn{5}{|c|}{ Exposition à vie à l'hépatite C (parmi les participants ayant fourni un échantillon de sang, $n=1044)^{3}$} \\
\hline Participants séropositifs pour l'hépatite $\mathrm{C}$ & $41,6 \%(434)$ & $46,1 \%(245)$ & $36,9 \%(189)$ & 0,003 \\
\hline \multicolumn{5}{|c|}{$\begin{array}{l}\text { État sérologique pour le VIH et l'hépatite C (parmi les participants qui ont fourni un échantillon de sang } \\
\text { suffisant pour effectuer le dépistage d'anticorps contre le VIH et l'hépatite } C, n=1044 \text { ) }\end{array}$} \\
\hline Participants séropositifs pour le VIH seulement & $0 \%(0)$ & $0 \%(0)$ & $0 \%(0)$ & \multirow{4}{*}{0,011} \\
\hline $\begin{array}{r}\text { Participants séropositifs pour l'hépatite C } \\
\text { seulement }\end{array}$ & $36,5 \%(381)$ & $40,2 \%(214)$ & $32,6 \%(167)$ & \\
\hline $\begin{array}{r}\text { Participants séropositifs pour le VIH et } \\
\text { I'hépatite C }\end{array}$ & $5,1 \%(53)$ & $5,8 \%(31)$ & $4,3 \%(22)$ & \\
\hline $\begin{array}{r}\text { Participants séronégatifs pour le VIH et } \\
\text { I'hépatite C }\end{array}$ & $58,4 \%(610)$ & $54 \%(287)$ & $63,1 \%(323)$ & \\
\hline \multicolumn{5}{|c|}{ Exposition à vie à la syphilis (parmi les participants qui ont fourni un échantillon de sang, $n=1045)^{4}$} \\
\hline Participants séropositifs pour la syphilis & $0,2 \%(2)$ & $0,2 \%(1)$ & $0,2 \%(1)$ & $0,977^{5}$ \\
\hline
\end{tabular}

\footnotetext{
${ }^{T}$ Le dépistage du VIH grâce à des échantillons de gouttes de sang séchées a été réalisé en utilisant la méthode AVIOQ HIV-1 EIA. Des tests de confirmation Western Blot ont ensuite été réalisés (Bio-Rad GS HIV-1). Un résultat positif indiquait une infection au VIH.

${ }^{2}$ Les participants qui ont indiqué que les derniers résultats de leur test de dépistage du VIH étaient positifs et qui ont été identifiés comme étant séropositifs pour le VIH d'après l'analyse de l'échantillon biologique fourni au moment de l'entrevue ont été classés comme étant au courant de leur séropositivité pour le VIH.

${ }^{3}$ Le dépistage de l'hépatite $\mathrm{C}$ grâce à des échantillons de sang séché a été réalisé en utilisant la version 3.0 du test VHC EIA Ortho ${ }^{\mathrm{MD}}$. Les tests de confirmation n'ont pas été effectués sur les échantillons dont le test de dépistage était positif. Un résultat positif indiquait une infection passée ou présente par l'hépatite $\mathrm{C}$, et ne permettait pas d'établir de distinction entre des infections aiguës, chroniques ou résolues.

${ }^{4}$ Le dépistage de la syphilis a été réalisé en utilisant l'essai de l'agglutination des particules Treponema pallidum (TP-PA) de Serodiaa. Les tests de confirmation n'ont pas été effectués sur les échantillons dont le test de dépistage était positif. Un résultat positif était dû à de faux résultats positifs ou à la présence d'anticorps contre la syphilis, ce qui indiquait une infection par la syphilis passée ou présente, mais ne permettait pas d'établir de distinction entre une infection aiguë et une infection chronique ou résolue.

${ }^{5}$ Il est à noter qu'en raison du faible nombre de résultats, les résultats du test du chi carré doivent être interprétés avec prudence.
}

Parmi les 1045 participants qui ont fourni un échantillon de sang suffisant pour le dépistage du VIH, 54 participants $(5,2 \%)$ ont été trouvés séropositifs pour le $\mathrm{VIH}$; il n'y avait aucune différence importante entre les hommes et les femmes (tableau 2). Parmi les 54 participants qui ont obtenu un résultat positif au test de dépistage du VIH, la majorité des femmes et des hommes avait des antécédents d'utilisation de drogues injectables; dans l'ensemble, 92,6\% de tous les participants séropositifs pour le VIH avaient, à un moment dans leur vie, utilisé des drogues injectables. Un peu plus de la moitié $(55,8 \%)$ des participants qui ont été trouvés séropositifs pour le VIH étaient au courant de leur séropositivité pour le $\mathrm{VIH}$; aucune différence importante n'a été relevée entre les hommes et les femmes.

II est intéressant de noter que la méconnaissance de l'état de l'infection au VIH était plus élevée chez les participants séropositifs ne présentant pas d'antécédents d'utilisation de drogues injectables. Parmi les 54 participants qui ont obtenu des résultats positifs au test du VIH, quatre d'entre eux ne s'étaient jamais injecté de drogues et la totalité de ceux-ci $(4 / 4 ; 100 \%)$ ignoraient leur séropositivité (données non représentées). Par opposition, parmi les 50 participants qui ont 
obtenu des résultats positifs au test du VIH et qui avaient des antécédents d'utilisation de drogues injectables, $42 \%$ (21/50) d'entre eux ignoraient leur séropositivité (données non représentées).

Parmi les 1044 participants qui ont fourni un échantillon suffisant pour le dépistage des anticorps de l'hépatite C, 41,6 \% étaient séropositifs; les proportions étaient beaucoup plus élevées chez les hommes que chez les femmes qui ont eu des résultats positifs au test de dépistage de l'exposition à l'hépatite $C$. Un résultat positif au dépistage de l'hépatite $C$ indique une infection passée ou présente par l'hépatite $C$, et ne permet pas d'établir de distinction entre des infections aiguës et des infections chroniques ou résolues. La séroprévalence de la syphilis était très faible chez les hommes et les femmes; dans l'ensemble, seulement $0,2 \%$ des participants étaient séropositifs pour la syphilis. Un résultat positif pour la syphilis indique une infection par la syphilis passée ou présente.

Bien qu'il ne soit pas possible de déterminer la proportion de participants qui étaient co-infectés par le VIH et l'hépatite C au moment de l'enquête en raison du test de laboratoire utilisé (c.-à-d. qu'il n'était pas possible de faire la distinction entre une infection passée ou présente par l'hépatite C), 5,1\% des participants ont été trouvés séropositifs pour le VIH et I'hépatite $\mathrm{C}$. Une proportion significativement plus élevée d'hommes que de femmes ont obtenu des résultats positifs au dépistage d'anticorps contre le VIH et l'hépatite C.

Tableau 3 : Comportements d'injection des participants à l'enquête pilote A-Track

\begin{tabular}{|c|c|c|c|c|}
\hline Comportement d'injection & Total & Hommes & Femmes & Valeur $p$ \\
\hline $\begin{array}{l}\text { Proportion de participants ayant déjà utilisé des } \\
\text { drogues injectables }(n=1063)\end{array}$ & $50 \%(532)$ & $53,4 \%(287)$ & $46,7 \%(245)$ & 0,029 \\
\hline $\begin{array}{l}\text { Proportion de participants ayant été trouvés } \\
\text { séropositifs pour le VIH d'après l'analyse de } \\
\text { l'échantillon biologique, parmi ceux qui ont signalé } \\
\text { avoir déjà utilisé des drogues injectables ( } n=528 \text { ) }\end{array}$ & $9,5 \%(50)$ & $10,2 \%(29)$ & $8,6 \%(21)$ & 0,549 \\
\hline $\begin{array}{l}\text { Proportion de participants dont la première } \\
\text { injection est survenue avant l'âge de } 16 \text { ans } \\
\text { ( } n=531)\end{array}$ & $19,4 \%(103)$ & $19,5 \%(56)$ & $19,3 \%(47)$ & 0,942 \\
\hline $\begin{array}{l}\text { Proportion de participants ayant utilisé des drogues } \\
\text { injectables dans les six mois précédant l'entrevue } \\
(n=1064)\end{array}$ & $30,3 \%(322)$ & $32,5 \%(175)$ & $28 \%(147)$ & 0,113 \\
\hline \multicolumn{5}{|c|}{$\begin{array}{l}\text { Comportements d'injection parmi les participants ayant indiqué avoir utilisé des drogues injectables dans les } \\
\text { six mois précédant l'entrevue }(n=322)\end{array}$} \\
\hline $\begin{array}{l}\text { Proportion de participants ayant utilisé des aiguilles } \\
\text { ou des seringues stériles lors de la dernière } \\
\text { injection }(n=321)^{1}\end{array}$ & $98,8 \%(317)$ & $97,7 \%(170)$ & $100 \%(147)$ & 0,064 \\
\hline $\begin{array}{l}\text { Proportion de participants ayant utilisé une aiguille } \\
\text { ou une seringue utilisée pour consommer des } \\
\text { drogues injectables au cours des six mois précédant } \\
\text { l'entrevue }(n=319)\end{array}$ & $9,1 \%(29)$ & $8,7 \%(15)$ & $9,6 \%(14)$ & 0,776 \\
\hline \multicolumn{5}{|l|}{$\begin{array}{l}\text { Drogues injectables utilisées les plus couramment } \\
\text { au cours des six mois précédant l'entrevue }{ }^{2}\end{array}$} \\
\hline Cocaïne & $56,7 \%(181)$ & $59,5 \%(103)$ & $53,4 \%(78)$ & 0,272 \\
\hline Morphine non prescrite & $51,1 \%(163)$ & $54,9 \%(95)$ & $46,6 \%(68)$ & 0,138 \\
\hline Ritalin & $49,8 \%(159)$ & $49,1 \%(89)$ & $50,7 \%(74)$ & 0,782 \\
\hline Talwin et Ritalin non prescrits & $17,2 \%(55)$ & $16,2 \%(28)$ & $18,5 \%(27)$ & 0,587 \\
\hline Dilaudid (hydromorphone) & $15,7 \%(50)$ & $15,6 \%(27)$ & $15,8 \%(23)$ & 0,971 \\
\hline $\begin{array}{l}\text { Personne la plus souvent signalée avec laquelle les } \\
\text { participants ont utilisé des drogues injectables dans }\end{array}$ & & & & \\
\hline
\end{tabular}




\begin{tabular}{|c|c|c|c|c|}
\hline \multicolumn{5}{|l|}{ les six mois précédant l'entrevue ${ }^{2}$} \\
\hline Amis ou personnes qu'ils connaissent bien & $53,3 \%$ (171) & $55,2 \%(96)$ & 51 \% (75) & 0,458 \\
\hline Partenaires sexuels réguliers & $47,7 \%(153)$ & $47,7 \%(83)$ & $47,6 \%(70)$ & 0,988 \\
\hline Injection pendant qu'ils étaient seuls & $40,2 \%(129)$ & $42 \%(73)$ & $38,1 \%(56)$ & 0,482 \\
\hline \multicolumn{5}{|l|}{$\begin{array}{l}\text { Endroit de l'injection le plus souvent signalé au } \\
\text { cours des six mois précédant l'entrevue }\end{array}$} \\
\hline Son propre appartement ou maison & $72,6 \%(233)$ & $77 \%(134)$ & $67,4 \%(99)$ & 0,053 \\
\hline Chez des amis & $44,2 \%(142)$ & $46,6 \%(81)$ & $41,5 \%(61)$ & 0,364 \\
\hline $\begin{array}{r}\text { Maison ou domicile d'un autre membre de sa } \\
\text { famille }\end{array}$ & $20,9 \%(67)$ & $20,7 \%(36)$ & $21,1 \%(31)$ & 0,930 \\
\hline
\end{tabular}

${ }^{1}$ Selon les obligations de déclaration internationales par l'entremise du Rapport d'activité sur la riposte au sida dans le monde, même si l'indicateur de ce Rapport est basé sur les répondants qui ont signalé l'utilisation de drogues injectables dans le dernier mois au lieu des six derniers mois.

${ }^{2}$ On a remis aux participants une liste de réponses et on leur a demandé de cocher toutes les réponses qui s'appliquaient à eux. Étant donné que les participants pouvaient choisir plus d'une réponse, le dénominateur total n'est pas présenté.

La moitié de tous les participants (50\%) ont signalé avoir utilisé, à un moment dans leur vie, des drogues injectables; une proportion beaucoup plus élevée d'hommes a signalé des antécédents d'utilisation de drogues injectables (tableau 3). Parmi les participants ayant déclaré s'être injecté des drogues à un moment donné, 9,5\% ont été trouvés séropositifs d'après l'analyse des échantillons biologiques au moment de l'entrevue. Un peu moins d'un cinquième (19,4 \%) des participants qui s'étaient déjà injecté des drogues ont indiqué l'avoir fait pour la première fois avant l'âge de 16 ans; aucune différence importante n'a été relevée entre les hommes et les femmes. Dans l'ensemble, près d'un tiers de tous les participants (30,3\%) avaient utilisé des drogues injectables pendant les six mois précédant l'entrevue; aucune différence importante n'a été observée entre les hommes et les femmes.

Parmi les 322 individus qui ont indiqué avoir utilisé des drogues injectables pendant les six mois précédant l'entrevue, la majorité $(98,8 \%)$ avait utilisé une aiguille ou une seringue propre lors de leur dernière injection; les proportions étaient semblables chez les hommes et les femmes. Toutefois, près d'un dixième $(9,1 \%)$ avaient utilisé une aiguille ou une seringue contaminée dans les six mois précédant l'entrevue, et aucune différence importante n'a été relevée entre les hommes et les femmes. En ce qui concerne les drogues ou les substances les plus souvent signalées comme ayant été injectées pendant les six mois précédant l'entrevue, aucune différence importante n'a été relevée entre les hommes et les femmes. La cocaïne ainsi que la morphine et le Ritalin non prescrits étaient les trois drogues les plus souvent utilisées par les hommes et les femmes. Aucune différence importante n'a été relevée entre les hommes et les femmes en ce qui concerne les personnes avec lesquelles ils s'injectent des drogues le plus souvent; parmi les hommes et les femmes, la catégorie « amis ou personnes qu'ils connaissent bien » et ensuite la catégorie " partenaires sexuels réguliers » étaient les plus souvent signalées pour décrire les personnes avec lesquelles ils s'injectaient. Chez les hommes et chez les femmes, leur propre appartement ou maison était l'emplacement de l'injection le plus souvent signalé dans les six mois précédant l'entrevue.

Plusieurs des indicateurs de comportement sexuel énumérés dans le tableau ci-dessous sont conformes aux obligations de déclaration internationales, notamment le Rapport d'activité sur la riposte au sida dans le monde (RARSM). Se reporter aux notes de bas de page pour savoir quels indicateurs sont conformes au Rapport d'activité sur la riposte au sida dans le monde.

Tableau 4 : Comportements sexuels des participants à l'enquête pilote A-Track

\begin{tabular}{|l|c|c|c|c|}
\hline \multicolumn{1}{|c|}{ Comportements sexuels } & Total & Hommes & Femmes & Valeur $\mathbf{p}$ \\
\hline $\begin{array}{l}\text { Proportion de participants ayant eu leurs premiers } \\
\text { rapports sexuels avant l'âge de 15 ans (parmi les } \\
\text { participants âgés de 16 à 24 ans, } \mathbf{n}=\mathbf{2 6 6} \text { ) }\end{array}$ & $41 \%(109)$ & $50 \%(58)$ & $34 \%(51)$ & 0,009 \\
\hline $\begin{array}{l}\text { Proportion de participants ayant eu plus d'un } \\
\text { partenaire sexuel au cours des 12 mois précédant } \\
\text { l'entrevue (n = 926) }\end{array}$ & $\begin{array}{c}42,7 \% \\
(395)\end{array}$ & $\begin{array}{c}45,5 \% \\
(210)\end{array}$ & $\begin{array}{l}39,9 \% \\
(185)\end{array}$ & 0,086 \\
\hline Proportion de participants ayant utilisé un & $52,7 \%$ & $57,7 \%$ & $47 \%(87)$ & 0,035 \\
\hline
\end{tabular}




\begin{tabular}{|l|l|l|l|l|}
\hline $\begin{array}{l}\text { préservatif lors de leur dernière relation sexuelle } \\
\text { (parmi les participants âgés de } \mathbf{1 6} \text { à } \mathbf{4 9} \text { ans qui ont } \\
\text { indiqué avoir eu plus d'un partenaire sexuel au cours } \\
\text { des } \mathbf{1 2} \text { mois précédant l'entrevue, } \mathbf{n}=\mathbf{3 9 3})^{\mathbf{1}}\end{array}$ & $(207)$ & $(120)$ & & \\
\hline $\begin{array}{l}\text { Proportion de participants ayant eu un partenaire- } \\
\text { client dans les } \mathbf{1 2} \text { mois précédant l'entrevue }(\mathbf{n}=\mathbf{8 7 6 )} \\
\mathbf{3}\end{array}$ & $7,2 \%(63)$ & $3,1 \%(14)$ & $11,4 \%(49)$ & $<0,001$ \\
\hline $\begin{array}{l}\text { Proportion de participants ayant utilisé un } \\
\text { préservatif lors de leur dernière relation sexuelle } \\
\text { avec un partenaire-client (parmi les participants } \\
\text { ayant signalé avoir eu un partenaire-client dans les } \\
\mathbf{1 2} \text { mois précédant l'entrevue, } \mathbf{n}=\mathbf{6 2} \text { ) }\end{array}$ & $82,3 \%(51)$ & $78,6 \%(11)$ & $83,3 \%(40)$ & $0,682^{4}$ \\
\hline
\end{tabular}

${ }^{1}$ Indicateur pour le Rapport d'activité sur la riposte au sida dans le monde.

${ }^{2}$ Cette mesure découle des réponses des participants à une série de questions liées au nombre de partenaires sexuels masculins réguliers, de partenaires sexuels masculins occasionnels, de partenaires sexuels féminins réguliers, de partenaires sexuels féminins occasionnels, de partenaires-clients et de partenaires sexuels rémunérés; seuls les participants qui ont fourni des réponses valides à au moins une des questions dans la série ont été inclus dans le dénominateur.

${ }^{3}$ Un partenaire-client est quelqu'un qui a échangé de l'argent, des drogues, des biens ou toute autre chose contre des relations sexuelles avec le participant.

${ }^{4}$ Il est à noter qu'en raison du faible nombre de résultats, les résultats du test du chi carré doivent être interprétés avec prudence.

Il a été constaté qu'une proportion beaucoup plus élevée d'hommes que de femmes entre l'âge de 16 et 24 ans ont eu leur première relation sexuelle avant l'âge de 15 ans (50\% des hommes par rapport à $34 \%$ des femmes) (tableau 4). Un peu moins de la moitié $(42,7 \%)$ de tous les participants ont signalé avoir eu plus d'un partenaire sexuel dans les 12 mois précédant l'entrevue; les proportions étaient semblables chez les hommes et chez les femmes. Parmi les participants âgés de 16 à 49 ans qui ont signalé avoir eu plus d'un partenaire dans les 12 mois précédant l'entrevue, une proportion beaucoup plus élevée d'hommes avaient utilisé un préservatif lors de leur dernière relation sexuelle $(57,7 \%$ des hommes par rapport à $47 \%$ des femmes). Parmi les participants ayant déclaré avoir eu un partenaire-client dans les 12 mois précédant l'entrevue, $82,3 \%$ ont signalé avoir utilisé un préservatif lors de leur dernière relation sexuelle; aucune différence importante n'a été relevée entre les hommes et les femmes.

Tableau 5 : Test de dépistage, soins et traitement liés au VIH des participants à l'enquête pilote A-Track

\begin{tabular}{|c|c|c|c|c|}
\hline Test de dépistage, soins et traitement liés au VIH & Total & Hommes & Femmes & Valeur $p$ \\
\hline $\begin{array}{l}\text { Proportion de participants ayant déjà subi un test de } \\
\text { dépistage du VIH ( } n=1049)\end{array}$ & $\begin{array}{c}71,5 \% \\
(750)\end{array}$ & $\begin{array}{c}67,7 \% \\
(360)\end{array}$ & $\begin{array}{c}75,4 \% \\
(390)\end{array}$ & 0,005 \\
\hline $\begin{array}{l}\text { Proportion de participants ayant subi un test de } \\
\text { dépistage du VIH dans les } 12 \text { mois précédant } \\
\text { l'entrevue (parmi les participants ayant déjà subi un } \\
\text { test de dépistage du VIH, } n=750 \text { ) }\end{array}$ & $\begin{array}{c}67,6 \% \\
(507)\end{array}$ & $\begin{array}{c}68,9 \% \\
(248)\end{array}$ & $\begin{array}{c}66,4 \% \\
(259)\end{array}$ & 0,469 \\
\hline $\begin{array}{l}\text { Proportion de participants ayant déclaré qu'ils sont } \\
\text { actuellement suivis par un médecin en raison de leur } \\
\text { VIH (parmi les participants ayant indiqué être } \\
\text { atteints du VIH, } n=30)^{1}\end{array}$ & $86,7 \%(26)$ & $80 \%(12)$ & $93,3 \%(14)$ & $0,283^{2}$ \\
\hline $\begin{array}{l}\text { Proportion de participants ayant déjà pris des } \\
\text { médicaments prescrits pour leur infection au VIH } \\
\text { (parmi les participants ayant indiqué être atteints du } \\
\text { VIH, } n=30 \text { ) }\end{array}$ & $66,7 \%(20)$ & $73,3 \%(11)$ & $60 \%(9)$ & 0,439 \\
\hline
\end{tabular}

${ }^{1}$ Cela signifie une seule visite, ou plus, chez un médecin ou un autre professionnel de la santé au cours des six mois précédant l'entrevue aux fins de dépistage du VIH, de traitements, de counseling, etc.

${ }^{2}$ Il est à noter qu'en raison du faible nombre de résultats, les résultats du test du chi carré doivent être interprétés avec prudence. 
Un peu moins de trois quarts $(71,5 \%)$ des participants ont signalé avoir subi un test de dépistage du VIH au moins une fois dans leur vie; les antécédents de dépistage du VIH étaient beaucoup plus élevés chez les femmes que chez les hommes (tableau 5). Parmi les 750 participants qui avaient subi un test de dépistage du $\mathrm{VIH}, 67,6 \%$ avaient subi un test pendant les 12 mois précédant l'entrevue; les proportions étaient semblables chez les hommes et les femmes. Parmi les participants ayant déclaré être séropositifs pour le $\mathrm{VIH}, 86,7 \%$ ont signalé être suivis par un médecin au moment de l'entrevue, et 66,7 \% ont indiqué avoir à un moment ou un autre pris des médicaments prescrits pour le VIH. Aucune différence importante n'a été relevée entre les hommes et les femmes.

\section{Conclusion}

Les résultats de l'enquête pilote A-Track sont conformes aux autres résultats qui semblent indiquer que les populations autochtones au Canada sont touchées de façon disproportionnée par le VIH (7-15). Ces résultats laissent également entendre que de nombreux comportements à risque peuvent contribuer à la transmission du VIH et à d'autres infections transmissibles par le sang parmi les populations autochtones, et soulignent donc le besoin continu de services de santé et de soutien social, ainsi que des tests de dépistage du VIH et d'autres infections transmissibles par le sang. Une analyse portant sur les déterminants sociaux de la santé des Autochtones permettrait d'améliorer davantage l'élaboration et la prestation des services en contextualisant les environnements de risque et de résilience qui ont une influence sur les différents comportements.

II existe toutefois des limites aux résultats. L'enquête pilote comprenait seulement des participants autochtones recrutés à des centres communautaires et de soins de santé à Regina. Par conséquent, il est possible que les résultats ne soient pas représentatifs de tous les Autochtones à Regina, ou de tous les Autochtones au Canada. De plus, les résultats de l'enquête pilote pour le système A-Track sont basés sur des données autodéclarées; il est donc possible que certains comportements à risque aient été surreprésentés ou sous-représentés. Malgré ces limites, les résultats de l'enquête pilote A-Track, la première en son genre au Canada, fournissent des renseignements précieux concernant les services de traitement et de prévention ainsi que les programmes offerts à l'échelle locale, provinciale et nationale. Ces données de surveillance peuvent être utilisées pour orienter les interventions existantes et concevoir de nouvelles stratégies visant à diminuer le risque d'infections au VIH et d'infections connexes parmi les populations autochtones au Canada.

En conclusion, cette évaluation des processus effectués et des résultats obtenus dans le cadre de l'élaboration et de la mise en œuvre de l'enquête pilote a démontré la faisabilité d'un tel système de surveillance comportementale dans les milieux urbains au Canada et permet de tirer des leçons aux fins d'utilisation lors de futures enquêtes du même genre.

\section{Remerciements}

L'enquête pilote A-Track menée à Regina était un partenariat du réseau All Nations Hope Network, de la région sanitaire de Regina Qu'Appelle, de l'Université des Premières nations du Canada, du Réseau canadien autochtone du sida et de l'Agence de la santé publique du Canada. Nous tenons à reconnaître et à souligner les contributions des participants à l'enquête A-Track, des membres des équipes d'enquête, des lieux d'enquête, des membres du comité consultatif communautaire et l'équipe chargée de l'enquête pilote A-Track (Margaret Poitras [chercheuse principale], Maurice Hennink [chercheur principal], Kathy Lloyd, Rachel Faye, Zahid Abbas, Carrie Bourassa, Renée Masching, Jill Tarasuk, Susanna Ogunnaike-Cooke [chercheuse principale], Chris Archibald). Nous tenons à souligner le soutien des laboratoires nationaux du VIH et de rétrovirologie (Paul Sandstrom, John Kim, Laurie Malloch, Richard Pilon), du Laboratoire national de microbiologie (Raymond Tsang) et des membres de l'équipe de surveillance A-Track de l'Agence de la santé publique du Canada (Chris Archibald, Susanna Ogunnaike-Cooke, Jill Tarasuk, Rachel Bennett, Rachel MacLean, Stephen Cule).

\section{Conflit d'intérêts}

Aucun

\section{Financement}

Ce travail a été appuyé par l'Agence de la santé publique du Canada. 


\section{Références}

(1) Public Health Agency of Canada. Summary: Estimates of HIV prevalence and incidence in Canada, 2011. Ottawa: PHAC; 2012.

(2) Statistics Canada. Population and dwelling count highlight tables, 2011 Census. Ottawa: Statistics Canada; 2012. http://www12.statcan.gc.ca/census-recensement/2011/dp-pd/hlt-fst/pd-pl/index-fr.cfm

(3) Public Health Agency of Canada. Summary of key findings from the A-Track pilot survey, 2011-2012. Ottawa: PHAC; 2014 http://www.phac-aspc.gc.ca/aids-sida/publication/reports/a-track/index-fr.php

(4) First Nations Centre. OCAP: Ownership, control, access and possession. Sanctioned by the First Nations Information Governance Committee, Assembly of First Nations. Ottawa: National Aboriginal Health Organization; 2007.

(5) Tri-Council policy statement: Ethical conduct for research involving humans. Canadian Institutes of Health Research, Natural Sciences and Engineering Research Council of Canada, and Social Sciences and Humanities Research Council of Canada; December 2010.

(6) Canadian Institutes for Health Research. Guidelines for health research involving Aboriginal people. Ottawa: Canadian Institutes of Health Research; 2007.

(7) Sullivan A, Swantee C, Rank C, Liu J, et al. Race/ethnicity of newly diagnosed HIV cases in Ontario, 2009 to 2011.2011 Ontario HIV Treatment Network Research Conference. Toronto, Ontario. November 14, 2011 (Abstract 138). http://conference.ohtn.on.ca/past-conferences/2011/Presentations/138.php

(8) Becker ML, Kasper K, Pindera C, et al. Characterizing the HIV epidemic in the prairie provinces. Can J Infect Dis Med Microbiol. 2012; 23(1):19-22.

(9) Becker M, Kasper K, Pindera C, Cheang M, et al. Evolution of the HIV epidemic in the prairie provinces. Can J Infect Dis Med Microbiol. 2010; Summer; 21(Suppl B):21B (Abstract O043).

(10) Rank C, Bangura H, Gilbert M, et al. Distribution and correlates of recent HIV infection in four Canadian provinces, $2000-2008$. Can J Infect Dis Med Microbiol. 2012; Spring;23(Suppl A): 86A (Abstract P148).

(11) Halverson J, Shea B, Andersson N, et al. Unequal burden: comparison of HIV diagnosis rates among Aboriginal and Caucasian populations in Canada. Can J Infect Dis Med Microbiol. 2011; Spring;22(Suppl B): 31B (Abstract O072).

(12) Khan I, Smith LA, Bukassa Kazadi G, et al. Know your status: outcomes of a community-based multi-disciplinary HIV testing and care project in a Saskatchewan First Nations community. Can J Infect Dis Med Microbiol. 2012; Spring;23(Suppl A):77A (Abstract P116).

(13) Oviedo-Joekes E, Christian WM, Pearce ME, Schechter MT, Spittal PM. The Cedar Project: vulnerabilities associated with HIV incidence among young Aboriginal people who use injection and non-injection drugs in two Canadian cities. Can $\mathrm{J}$ Infect Dis Med Microbiol. 2011; Spring;22(Suppl B):30B-31B (Abstract O070).

(14) Palmer A, Forrest JI, Wang $\mathrm{H}$, et al. Cohort profile: longitudinal investigations into supportive and ancillary health services (LISA). Can J Infect Dis Med Microbiol. 2011; Spring;22(Suppl B):93B-94B (Abstract P186).

(15) Marshall C, Lalonde K, Halverson J, Archibald C. An older, changing face: HIVIAIDS trends among Canadians 50 years of age and older. Can J Infect Dis Med Microbiol. 2010; Summer;21(Suppl B):66B (Abstract P199). 